\title{
ON PARALLELIZABILITY AND SPAN OF THE DOLD MANIFOLDS
}

\author{
JÚLIUS KORBA
}

(Communicated by Daniel Ruberman)

\begin{abstract}
The Dold manifold $P(m, n)$ is obtained from the product $S^{m} \times$ $\mathbb{C} P^{n}$ of the $m$-dimensional sphere and $n$-dimensional complex projective space by identifying $\left(x,\left[z_{1}, \ldots, z_{n+1}\right]\right)$ with $\left(-x,\left[\bar{z}_{1}, \ldots, \bar{z}_{n+1}\right]\right)$, where $\bar{z}$ denotes the complex conjugate of $z$. We answer the parallelizability question for the Dold manifolds $P(m, n)$ and, by completing an earlier (2008) result due to Peter Novotný, we solve the vector field problem for the manifolds $P(m, 1)$.
\end{abstract}

\section{INTRODUCTION AND PRELIMINARIES}

Given a finite-dimensional real vector bundle $\alpha$ over a CW-complex, its span, denoted $\operatorname{span}(\alpha)$, is defined to be the maximum number of its everywhere linearly independent cross-sections. To fix some further notation, $k \alpha$ will always denote the $k$-fold Whitney sum $\alpha \oplus \cdots \oplus \alpha$, and the letter $\varepsilon$ will be reserved for denoting the trivial line bundle. Note that $\operatorname{span}(\alpha) \geq k$ if and only if $\alpha \approx \sigma \oplus k \varepsilon$ for some vector bundle $\sigma$.

For a $q$-dimensional smooth connected manifold $M^{q}$ one defines its span, denoted $\operatorname{span}(M)$, to be $\operatorname{span}(T M)$, where $T M$ is the tangent bundle of $M$. The manifold $M^{q}$ is parallelizable if $\operatorname{span}(M)=q$. The problem of determining $\operatorname{span}(M)$ is known as the vector field problem on $M$. By the Hopf theorem on vector fields, a smooth closed connected manifold has span at least one if and only if its Euler-Poincaré characteristic vanishes. For further information, we refer to [7, [8, [9, [17.

In addition to the span of a manifold $M$, one defines its stable span ([7, 8], [9]), denoted stable $\operatorname{span}(M)$, to be the integer $\operatorname{span}(T M \oplus \varepsilon)-1$. Note that the geometric dimension of a real vector bundle $\alpha$ is defined to be the smallest $g$ such that there exists a $g$-dimensional real vector bundle which is stably isomorphic to $\alpha$ (recall that two vector bundles are stably isomorphic if their sums with suitable trivial bundles are isomorphic). In this terminology, stable $\operatorname{span}\left(M^{q}\right)=k$ means that the geometric dimension of the (tangent bundle of the) manifold $M^{q}$ is $q-k$. For the question of how stable span and span are related, as well as for several topics where the knowledge of these invariants can be useful, see, for instance, [7.

Received by the editors November 10, 2011.

2010 Mathematics Subject Classification. Primary 57R25; Secondary 55S40, 57R20.

Key words and phrases. Dold manifold, vector field problem, span, stable span, parallelizable manifold, stably parallelizable manifold.

Part of this research was carried out while the author was a member of two research teams supported in part by the grant agency VEGA (Slovakia). 
Stably parallelizable manifolds (also called $\pi$-manifolds by some authors) are those for which the stable span and dimension coincide. For instance, each $n$ sphere $S^{n}(n \geq 1)$ is stably parallelizable. But ([2]) among them only $S^{1}, S^{3}$, and $S^{7}$ are parallelizable.

The central objects of this paper, the Dold manifolds, were introduced by Albrecht Dold in [4] in order to concretize odd-dimensional generators for the unoriented cobordism ring. The Dold manifold $P(m, n)(m \geq 0, n \geq 0, m+n>0)$ is a smooth closed connected manifold of dimension $m+2 n$, obtained from the product $S^{m} \times \mathbb{C} P^{n}$ of the $m$-dimensional sphere and $n$-dimensional complex projective space by identifying $\left(x,\left[z_{1}, \ldots, z_{n+1}\right]\right)$ with $\left(-x,\left[\bar{z}_{1}, \ldots, \bar{z}_{n+1}\right]\right)$, where $\bar{z}$ denotes the complex conjugate of $z$. In particular, $P(m, 0)$ is the $m$-dimensional real projective space $\mathbb{R} P^{m}$ and $P(0, n)$ is $\mathbb{C} P^{n}$. For further information and details about this family of manifolds, in addition to [4, we recommend [14, [15, [16], [18.

Over $P(m, n)$, there exists a real line bundle $\xi$ (for $P(m, 0)$, this is the Hopf line bundle $\xi_{m}$ over $\left.\mathbb{R} P^{m}\right)$, and we also have a 2 -dimensional real vector bundle $\eta$ over $P(m, n)$ (for $P(0, n)$, this is the realification of the canonical complex line bundle over $\left.\mathbb{C} P^{n}\right)$; we refer to 18 for the basic properties of $\xi$ and $\eta$. The $\mathbb{Z}_{2}$-cohomology ring of $P(m, n)$ is isomorphic to a quotient ring,

$$
H^{*}\left(P(m, n) ; \mathbb{Z}_{2}\right) \cong \mathbb{Z}_{2}[u, v] /\left(u^{m+1}=0, v^{n+1}=0\right),
$$

where $u \in H^{1}\left(P(m, n) ; \mathbb{Z}_{2}\right) \cong \mathbb{Z}_{2}, v \in H^{2}\left(P(m, n) ; \mathbb{Z}_{2}\right)$, and for the Stiefel-Whitney classes of $\xi$ and $\eta$ we have $w_{1}(\xi)=u, w_{1}(\eta)=u$ and $w_{2}(\eta)=v$.

Standard properties of vector bundles ( 6 ) enable one to cancel one copy of $\xi$ in Ucci's description ([18, Theorem 1.5]) of $T P(m, n)$. So one obtains the following formula for the stable tangent bundle of $P(m, n)$ :

$$
T P(m, n) \oplus 2 \varepsilon \approx m \xi \oplus(n+1) \eta .
$$

From this, for the total Stiefel-Whitney class one has

$$
w(P(m, n))=(1+u)^{m}(1+u+v)^{n+1} .
$$

We have the following aims in this paper. In Section 2, we derive an upper bound (18) below) for the stable span of the Dold manifolds $P(m, n)$. In Section 3, we use (18) for answering the parallelizability question for all $P(m, n)$. Finally, in Section 4, we apply (8) to determine the span of the manifolds $P(m, 1)$, including those having $m \equiv 15(\bmod 16)$. The latter manifolds were not covered by Novotný's results in [15, Section 4].

\section{An UPPER BOUND FOR stable $\operatorname{span}(P(m, n))$}

The following stability lemma is known. Therefore we omit its proof and will also use other standard facts about stability of vector bundles that can be found for instance in [6].

Lemma 2.1. Let $\alpha$ be a real $k$-dimensional vector bundle over a $C W$-complex of dimension $d$, and let $k>d$. Then $\operatorname{span}(\alpha) \geq k-d$, and for all $q \geq 0$ one has that

$$
\operatorname{span}(\alpha \oplus q \varepsilon)=q+\operatorname{span}(\alpha) .
$$

Now let $i: \mathbb{R} P^{m} \rightarrow P(m, n)$ be the obvious inclusion, so that for the pullback we have $i^{*}(\xi) \approx \xi_{m}$. In addition ([18, Proposition 1.4]), $i^{*}(\eta) \approx \varepsilon \oplus \xi_{m}$. From (2), it follows that

$$
i^{*}(T P(m, n)) \oplus 2 \varepsilon \approx(m+n+1) \xi_{m} \oplus(n+1) \varepsilon
$$


and by cancelling (for stability reasons) one copy of $\varepsilon$, we arrive at

$$
i^{*}(T P(m, n) \oplus \varepsilon) \approx(m+n+1) \xi_{m} \oplus n \varepsilon .
$$

The isomorphism (5) readily implies that

$$
\operatorname{span}\left((m+n+1) \xi_{m} \oplus n \varepsilon\right) \geq \operatorname{span}(T P(m, n) \oplus \varepsilon) .
$$

Since $\operatorname{span}(T P(m, n) \oplus \varepsilon)=1+$ stable $\operatorname{span}(P(m, n))$, we obtain that

$$
\text { stable } \operatorname{span}(P(m, n)) \leq \operatorname{span}\left((m+n+1) \xi_{m} \oplus n \varepsilon\right)-1 .
$$

This and Lemma 2.1 immediately imply the following:

$$
\text { stable } \operatorname{span}(P(m, n)) \leq n-1+\operatorname{span}\left((m+n+1) \xi_{m}\right) \text {. }
$$

This relates the (stable) span problem for the Dold manifolds $P(m, n)$ to the generalized vector field problem, the latter being the question of determining the span of any multiple of $\xi_{m}$ over $\mathbb{R} P^{m}$, for any $m$. Its solution is not yet completely known, but one knows it in many cases; see, for example, [3, [10, [11. The inequality (8) will serve as a basis for solving the parallelizability question for the Dold manifolds $P(m, n)$ in Section 3 as well as for completing the determination of the span for the Dold manifolds $P(m, 1)$ in Section 4 .

Remark 2.1. For $n=0$, formula (5) is nothing but the well-known description ([13, Theorem 4.5]) of the stable tangent bundle of $\mathbb{R} P^{m}$, and in this case (8) turns into equality. In Section (4) we shall prove that (8) also turns into equality for the manifolds $P(m, 1)$ if $m$ is odd.

\section{The parallelizability Problem for $P(m, n)$}

The parallelizability and stable parallelizability questions for the Dold manifolds seem to be open up to now. We answer them in the following theorem.

Theorem 3.1. The Dold manifold $P(m, n)$ (for $m \geq 0, n \geq 0, m+n>0$ ) is stably parallelizable if and only if

$$
(m, n) \in\{(1,0),(3,0),(7,0),(0,1),(2,1),(6,1)\} .
$$

The manifolds $P(1,0) \cong \mathbb{R} P^{1}, P(3,0) \cong \mathbb{R} P^{3}$, and $P(7,0) \cong \mathbb{R} P^{7}$ are the parallelizable real projective spaces. None of the remaining stably parallelizable Dold manifolds, $P(0,1) \cong S^{2}, P(2,1)$, and $P(6,1)$, are parallelizable.

Proof. In inequality (8), notice that $n-1+\operatorname{span}\left((m+n+1) \xi_{m}\right) \leq n-1+\operatorname{span}((m+$ $n+1) \varepsilon)=n-1+m+n+1=m+2 n=\operatorname{dim}(P(m, n))$. This means that whenever we know that the vector bundle $(m+n+1) \xi_{m}$ is not trivial, we immediately know that the manifold $P(m, n)$ is not stably parallelizable. We use this idea in our proof, but it turns out that it suffices to apply it to the Dold manifolds $P(m, 0)$ and $P(m, 1)$. Indeed, if the manifold $P(m, n)$ is stably parallelizable, then its double cover $S^{m} \times \mathbb{C} P^{n}$ is stably parallelizable as well. But the latter is stably parallelizable precisely when $n \in\{0,1\}$, as we now show. Of course, the manifolds $S^{m} \times \mathbb{C} P^{0} \cong S^{m}$ and $S^{m} \times \mathbb{C} P^{1} \cong S^{m} \times S^{2}$ are stably parallelizable. For the remaining cases, we have the map $j: \mathbb{C} P^{n} \rightarrow S^{m} \times \mathbb{C} P^{n}, j(x)=\left(x_{0}, x\right)$ (where $\left.x_{0}=(1,0, \ldots, 0) \in S^{m}\right)$, such that $j^{*}\left(T\left(S^{m} \times \mathbb{C} P^{n}\right)\right) \approx m \varepsilon \oplus T \mathbb{C} P^{n}$. Thus, if the product $S^{m} \times \mathbb{C} P^{n}$ is stably parallelizable, then $\mathbb{C} P^{n}$ has the same property. But it is classical that the complex projective space $\mathbb{C} P^{n}$ is stably parallelizable only for $n \in\{0,1\}$. Indeed, the tangent bundle $T \mathbb{C} P^{n}$ can be identified with the 
realification $\tau \mathbb{C} P_{\mathbb{R}}^{n}$ of the complex tangent bundle $\tau \mathbb{C} P^{n}$, and (for example) by [13, 15.6], the first Pontrjagin class $p_{1}\left(\tau \mathbb{C} P_{\mathbb{R}}^{n}\right)=(n+1) a^{2} \in H^{4}\left(\mathbb{C} P^{n} ; \mathbb{Z}\right)$ does not vanish, for $n>1$, because $H^{*}\left(\mathbb{C} P^{n} ; \mathbb{Z}\right) \cong \mathbb{Z}[a] /\left(a^{n+1}\right)$, where $-a \in H^{2}\left(\mathbb{C} P^{n} ; \mathbb{Z}\right)$ is the first Chern class of the canonical complex line bundle over $\mathbb{C} P^{n}$.

So we are left with just $P(m, 0)$ and $P(m, 1)$. Of course, $P(m, 0) \cong \mathbb{R} P^{m}$, and so, as is well known (see [8] for more details), the only stably parallelizable (and the only parallelizable) manifolds $P(m, 0)(m>0)$ are $P(1,0), P(3,0)$, and $P(7,0)$.

It remains to consider the manifolds $P(m, 1)$. The inequality (8) now reads

$$
\text { stable } \operatorname{span}(P(m, 1)) \leq \operatorname{span}\left((m+2) \xi_{m}\right) \text {. }
$$

The theorem will therefore be proved if we verify that

$$
\operatorname{span}\left((m+2) \xi_{m}\right)<m+2
$$

for all $m \notin\{0,2,6\}$ and also show that $P(2,1)$ and $P(6,1)$ are stably parallelizable but not parallelizable (of course, $P(0,1) \cong S^{2}$ is stably parallelizable but not parallelizable).

If $m$ is odd, then $(m+2) \xi_{m}$ is nonorientable or, equivalently, $w_{1}\left((m+2) \xi_{m}\right) \neq 0$, and therefore $\operatorname{span}\left((m+2) \xi_{m}\right)<m+2$. If $m \geq 2$ is even, then $2^{k-1} \leq m<2^{k}$ for a unique $k, k \geq 2$. For $m \neq 2^{k}-2$ we have $2^{k-1}+2 \leq m+2 \leq 2^{k}-2$. Then the dyadic expansion of $m+2$ must contain $2^{k-1}$; thus $\left(\begin{array}{c}m+2 \\ 2^{k-1}\end{array}\right)$ is odd by Lucas's theorem. Therefore the Stiefel-Whitney class $w_{2^{k-1}}\left((m+2) \xi_{m}\right)$ does not vanish, and we have $\operatorname{span}\left((m+2) \xi_{m}\right)<m+2$. Now it remains to consider $m=2^{k}-2$ for $k \geq 2$. Let us define $\varphi(t)$ to be the number of integers $s$ such that $0<s \leq t$ and $s \equiv 0,1,2$ or 4 $(\bmod 8)$. Then by Adams's theorem ([1, Theorem 7.4]), the reduced real $K$-group $\tilde{K}_{\mathbb{R}}\left(\mathbb{R} P^{t}\right)$ is cyclic of order $2^{\varphi(t)}$ with generator $\xi_{t}-1$. According to this theorem, if $\operatorname{span}\left(2^{k} \xi_{2^{k}-2}\right)=2^{k}$, that is, if $2^{k} \xi_{2^{k}-2}$ is (stably) trivial, then $2^{\varphi\left(2^{k}-2\right)}=2^{2^{k-1}-1}$ divides $2^{k}$, and therefore $2^{k-1}-1 \leq k$. But the latter is easily seen to be true for $k \geq 2$ only if $k \in\{2,3\}$, hence only for $m \in\{2,6\}$. To complete the proof, we observe that, by Ucci's theorem ([18, Theorem 3.8]), the 4-dimensional orientable manifold $P(2,1)$ immerses in $\mathbb{R}^{5}$ and the 8-dimensional orientable manifold $P(6,1)$ immerses in $\mathbb{R}^{9}$; by Hirsch-Smale theory $([5])$, this is equivalent to their stable parallelizability. But none of $P(2,1)$ and $P(6,1)$ are parallelizable; for a reason, see the beginning of the proof of Theorem 4.1. The proof of Theorem 3.1]is finished.

Remark 3.1. The author's first proof of Theorem 3.1 was based on the StiefelWhitney classes and Davis's theorem ([3. Theorem 1.1.(i)]). In fact Adams's theorem could also cover those cases in the proof of Theorem 3.1, for which we employed the Stiefel-Whitney classes (of course, they are historically prior to $K$-theory). More precisely, according to Adams's theorem, $(m+2) \xi_{m}$ is (stably) trivial if and only if $2^{\varphi(m)}$ divides $m+2$. For $m \leq 8$ one easily checks that this happens for precisely $m=2,6$, and it never happens for $m>8$ since in this range it is easy to see that $m+2<2^{\varphi(m)}$.

Remark 3.2. One has (cf. Remark 2.1) $T \mathbb{R} P^{m} \oplus \varepsilon \approx(m+1) \xi_{m}$. This fact, stability properties of vector bundles, and Hirsch-Smale immersion theory ([5]) imply that, for even $m$, the vector bundle $(m+2) \xi_{m}$ is trivial if and only if $\mathbb{R} P^{m}$ immerses (with $\xi_{m}$ as a normal bundle) in $\mathbb{R}^{m+1}$. Since $\mathbb{R} P^{2}$ immerses in $\mathbb{R}^{3}$ and $\mathbb{R} P^{6}$ immerses in $\mathbb{R}^{7}$, our proof of Theorem 3.1 also shows that the only positive even numbers $m$ such that $\mathbb{R} P^{m}$ immerses in $\mathbb{R}^{m+1}$ are $m=2,6$ (cf. [12, p. 284]). 


\section{The span of the Dold manifolds $P(m, 1)$}

In his Ph.D. thesis (partially published in [15]), Novotný determined the number $\operatorname{span}(P(m, 1))$ for all $m \not \equiv 15(\bmod 16)$. We complete his results by proving the following.

Theorem 4.1. The Dold manifold $P(m, 1)$ has its span zero for all even $m$. For every odd $m$, we write $m+1=($ odd number $) \cdot 2^{c+4 d}$, where $c$ and $d$ are such that $0 \leq c \leq 3$ and $d \geq 0$. Then we have

$$
\operatorname{span}(P(m, 1))=1+\operatorname{span}\left(S^{m}\right)=2^{c}+8 d .
$$

Proof. If $m$ is even, then the Euler-Poincare characteristic $\chi(P(m, 1))=2 \neq 0$ (since the double cover $S^{m} \times \mathbb{C} P^{1}$ has the Euler-Poincaré characteristic equal to $4)$, and therefore $\operatorname{span}(P(m, 1))=0$.

Now let us suppose that $m$ is odd. Then Novotný ([15, Corollary 4.3]) says that $\operatorname{span}(P(m, 1)) \geq 1+\operatorname{span}\left(S^{m}\right)$; this conjoined with (8) gives that

$$
\operatorname{span}\left((m+2) \xi_{m}\right) \geq \operatorname{span}(P(m, 1)) \geq 2^{c}+8 d .
$$

Thus, for proving Theorem 4.1, it suffices to verify that we have

$$
\operatorname{span}\left((m+2) \xi_{m}\right) \leq 2^{c}+8 d
$$

for all odd $m$.

Cases $m=16 t+i, i=1,3,5,7,9,11,13$. In all these cases (which were already covered, in a different way, in [15]) we have $d=0$. Clearly, if $i=1,5,9,13$, then $c=1$; if $i=3,11$, then $c=2$; and if $i=7$, then $c=3$. To verify (11) is thus equivalent to showing that

$$
\operatorname{span}\left((m+2) \xi_{m}\right) \leq 2^{c}
$$

We see that if $c=1$, then

$$
w_{m}\left(\left((m+2) \xi_{m}\right)\right)=\left(\begin{array}{c}
m+2 \\
m
\end{array}\right) w_{1}(\xi)^{m} \neq 0 ;
$$

if $c=2$, then

$$
w_{m-2}\left(\left((m+2) \xi_{m}\right)\right)=\left(\begin{array}{c}
m+2 \\
m-2
\end{array}\right) w_{1}(\xi)^{m-2} \neq 0 ;
$$

and, finally, if $c=3$, then

$$
w_{m-6}\left(\left((m+2) \xi_{m}\right)\right)=\left(\begin{array}{l}
m+2 \\
m-6
\end{array}\right) w_{1}(\xi)^{m-6} \neq 0 .
$$

Thus (12) (and (11)) is verified for all $m \not \equiv 15(\bmod 16)$.

Now we pass to the cases which were left open in [15].

Cases $m=16 t+15$. For these values of $m$, Stiefel-Whitney classes are insufficient to establish the results (see also Remark 4.1). Instead, we shall use [3. Theorem 1.1(ii)] for verifying the inequality (11).

Since $\left(\begin{array}{l}16 t+18 \\ 16 t+16\end{array}\right)$ is odd, by [3, Theorem 1.1(ii)] we have that over $\mathbb{R} P^{16 t+15}$, $\operatorname{span}\left((16 t+17) \xi_{16 t+15}\right) \leq 2+2(c+4 d)+\epsilon_{2}(c+4 d, 16 t+17)=2+2 c+8 d+\epsilon_{2}(c, 1)$, where $\epsilon_{2}(0,1)=-1, \epsilon_{2}(1,1)=-2, \epsilon_{2}(2,1)=-2$, and $\epsilon_{2}(3,1)=0$. 
Hence if $c=0$, we have

$$
\operatorname{span}\left((16 t+17) \xi_{16 t+15}\right) \leq 2+8 d+\epsilon_{2}(0,1)=8 d+1=2^{c}+8 d ;
$$

if $c=1$, then

$$
\operatorname{span}\left((16 t+17) \xi_{16 t+15}\right) \leq 4+8 d+\epsilon_{2}(1,1)=4+8 d-2=8 d+2=2^{c}+8 d ;
$$

if $c=2$, then

$$
\operatorname{span}\left((16 t+17) \xi_{16 t+15}\right) \leq 6+8 d+\epsilon_{2}(2,1)=6+8 d-2=8 d+4=2^{c}+8 d ;
$$

and if $c=3$, then

$$
\operatorname{span}\left((16 t+17) \xi_{16 t+15}\right) \leq 8+8 d+\epsilon_{2}(3,1)=8+8 d=2^{c}+8 d .
$$

So we have verified (11) for all values of $c$, completing the proof of Theorem 4.1.

Remark 4.1. For $m \equiv 15(\bmod 16)$, the Stiefel-Whitney classes of $P(m, 1)$ are not "sufficiently strong" for deriving our solution of the vector field problem for $P(m, 1)$, presented in Theorem 4.1. Indeed, in the dyadic expansion, we now have $m+2=1+2^{r}+\sum_{j=r+1}^{s} \mu_{j} 2^{j}$, where $2^{r}(r \geq 4)$ is the biggest power of 2 dividing $m+1$. Using (3), one can calculate that the best upper estimate implied by the Stiefel-Whitney classes of $P(m, 1)$ is $\operatorname{span}(P(m, 1)) \leq 2^{r}$. Writing $r=c+4 d$, where $0 \leq c \leq 3$ and $d \geq 1$, one knows that $1+\operatorname{span}\left(S^{m}\right)=2^{c}+8 d$, and it is readily seen that $2^{c}+8 d<2^{c+4 d}=2^{r}$ for all $d \geq 1$. In other words, the lower bound $2^{c}+8 d$ for $\operatorname{span}(P(m, 1))$ given in 15 , is, for $m \equiv 15(\bmod 16)$, always smaller than the smallest upper bound $2^{r}$ implied by the highest non-vanishing StiefelWhitney class of $P(m, 1)$. For instance, if $m=2^{k+4}+15$ for some $k \geq 1$, then $r=4, c=0, d=1$; and by Theorem 4.1 we know that $\operatorname{span}(P(m, 1))=9$, while the best "Stiefel-Whitney" upper estimate is $\operatorname{span}(P(m, 1)) \leq 16$.

\section{ACKNOWLEDGEMENT}

The author thanks Peter Zvengrowski for useful comments related to both the mathematical content and the presentation of this paper, in particular for suggesting the short $K$-theoretic proof used in Theorem 3.1 (see Remark 3.1).

\section{REFERENCES}

[1] Adams, J.: Vector fields on spheres, Bull. Amer. Math. Soc. 68 (1962), 39-41. MR.0133837 (24:A3662)

[2] Bott, R., Milnor, J.: On the parallelizability of the spheres, Bull. Amer. Math. Soc. 64 (1958), 87-89. MR0102804 (21:1590)

[3] Davis, D.: Generalized homology and the generalized vector field problem, Quart. J. Math. Oxford Ser. (2) 25 (1974), 169-193. MR0356053 (50:8524)

[4] Dold, A.: Erzeugende der Thomschen Algebra $\mathcal{N}$, Math. Z. 65 (1956), 25-35. MR.0079269 (18:60c)

[5] Hirsch, M.: Immersions of manifolds, Trans. Amer. Math. Soc. 93 (1959), 242-276. MR0119214 (22:9980)

[6] Husemoller, D.: Fibre Bundles, Third Edition, Graduate Texts in Math., Springer, New York, 1994. MR1249482(94k:55001)

[7] Korbaš, J.: Distributions, vector distributions, and immersions of manifolds in Euclidean spaces, Chapter 13 (pp. 665-724) in: Handbook of Global Analysis (edited by D. Krupka and D. Saunders), Elsevier, Amsterdam, 2008. MR.2389644(2009g:58001)

[8] Korbaš, J., Zvengrowski, P.: The vector field problem: A survey with emphasis on specific manifolds, Exposition. Math. 12 (1994), 3-20. MR1267626 (94m:57054)

[9] Koschorke, U.: Vector Fields and Other Vector Bundle Morphisms - A Singularity Approach, Lecture Notes in Math. 847, Springer-Verlag, Berlin, 1981. MR611333 (82i:57026) 
[10] Lam, K. Y.: Sectioning vector bundles over real projective space, Quart. J. Math. Oxford (2) 23 (1972), 97-106. MR0296965 (45:6024)

[11] Lam, K. Y., Randall, D.: Geometric dimension of bundles on real projective spaces, Homotopy theory and its applications (Cocoyoc, 1993), 137-160, Contemp. Math. 188, Amer. Math. Soc., Providence, RI, 1995. MR.1349135 (97c:55030)

[12] Milnor, J.: On the immersion of $n$-manifolds in $(n+1)$-space, Comment. Math. Helv. 30 (1956), 275-284. MR0079268 (18:60b)

[13] Milnor, J., Stasheff, J.: Characteristic Classes, Ann. Math. Stud. 76, Princeton Univ. Press, Princeton, NJ, 1974. MR0440554 (55:13428)

[14] Mukerjee, H. K.: Classification of homotopy Dold manifolds, New York J. Math. 9 (2003), 271-293. MR2016190 (2004h:57040)

[15] Novotný, P.: Span of Dold manifolds, Bull. Belg. Math. Soc. Simon Stevin 15 (2008), 687-698. MR2475492(2010a:57047)

[16] Stong, R.: Vector bundles over Dold manifolds, Fund. Math. 169 (2001), 85-95. MR.1852354 (2002e:57036)

[17] Thomas, E.: Vector fields on manifolds, Bull. Amer. Math. Soc. 75 (1969), 643-683. MR.0242189(39:3522)

[18] Ucci, J.: Immersions and embeddings of Dold manifolds, Topology 4 (1965), 283-293. MR0187250(32:4703)

Department of Algebra, Geometry, and Mathematical Education, Faculty of Mathematics, Physics, and Informatics, Comenius University, Mlynská dolina, SK-842 48 Bratislava 4, Slovakia - and - Mathematical Institute, Slovak Academy of Sciences, Štefánikova 49, SK-814 73 Bratislava 1, Slovakia

E-mail address: korbas@fmph.uniba.sk 